\title{
Diversity of methanogens in the hindgut of captive white rhinoceroses, Ceratotherium simum
}

Yu-heng Luo ${ }^{1 *}$, André-Denis G Wright ${ }^{2}$, You-long Li ${ }^{3}$, Hua Li ${ }^{1}$, Qi-hong Yang ${ }^{4}$, Ling-juan Luo ${ }^{5}$ and Ming-xian Yang ${ }^{6}$

\begin{abstract}
Background: The white rhinoceros is on the verge of extinction with less than 20,200 animals remaining in the wild. In order to better protect these endangered animals, it is necessary to better understand their digestive physiology and nutritional requirements. The gut microbiota is nutritionally important for herbivorous animals. However, little is known about the microbial diversity in the gastrointestinal tract (GIT) of the white rhinoceros. Methanogen diversity in the GIT may be host species-specific and, or, function-dependent. To assess methanogen diversity in the hindgut of white rhinoceroses, an archaeal 16S rRNA gene clone library was constructed from pooled PCR products obtained from the feces of seven adult animals.

Results: Sequence analysis of 153 archaeal 16S rRNA sequences revealed 47 unique phylotypes, which were assigned to seven operational taxonomic units (OTUs 1 to 7). Sequences assigned to OTU-7 (64 out of 153 total sequencs - 42\%) and OTU-5 (18\%, 27/153) had $96.2 \%$ and $95.5 \%$ identity to Methanocorpusculum labreanum, respectively, making Methanocorpusculum labreanum the predominant phylotype in these white rhynoceroses. Sequences belonging to OTU-6 $(27 \%, 42 / 153)$ were related $(97.6 \%)$ to Methanobrevibacter smithii. Only $4 \%$ of the total sequences (6/153) were assigned to Methanosphaera stadtmanae (OTU-1). Sequences belonging to OTU-2 $(4 \%, 6 / 153)$, OTU-3 (3\%, 5/153) and OTU-4 (2\%, 3/153) were distantly related (87.5 to 88,4\%) to Methanomassiliicoccus luminyensis and were considered to be novel species or strains that have yet-to-be cultivated and characterized.

Conclusion: Phylogenetic analysis indicated that the methanogen species in the hindgut of white rhinoceroses were more similar to those in the hindgut of horses. Our findings may help develop studies on improving the digestibility of forage for sustainable management and better health of these endangered animals.
\end{abstract}

Keywords: White rhinoceros, Methanogen, Gut microbial diversity

\section{Background}

The white rhinoceros (Ceratotherium simum) belongs to the family Rhinocerotidae (order Perrisodactyla) and is the largest of the five species of rhinoceros and the world's third largest land mammal after the African and Indian elephants. It has a massive body and large head, and its weight ranges from 1,360 to 3,630 kg. White rhinoceroses are herbivore grazers. They spend about half of the day eating grass and are normally found in the savannah and grassland habitats [1]. These large oddtoed ungulates are hindgut colonic fermenters, so they

\footnotetext{
*Correspondence: luoluo212@126.com

'Key Laboratory for Animal Disease-Resistance Nutrition of China and Ministry of Education; Institute of Animal Nutrition, Sichuan Agricultural University, 625014, Ya'an, China

Full list of author information is available at the end of the article
}

typically have a proportionally longer large intestine than small intestine.

White rhinoceroses are well known for their two horns, which have resulted in many of these animals being killed by poachers for their horns. Now the white rhinoceros is on the International Union for Conservation of Nature and Natural Resources (IUCN) Red List of Threatened Species [2]. The white rhinoceros once roamed much of sub-Saharan Africa, but today is on the near threatened list with less than 20,200 of these animals remaining in the wild [2]. One of the prerequisites to better protect these endangered animal species is to better understand their digestive physiology and nutritional requirements. Given the importance of the gut microbiota in herbivorous animals, little is known about the hindgut microorganisms in the white rhinoceros.

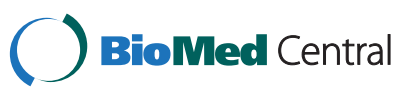


Methanogenic archaea, also called methanogens, exist widely in the GIT of many vertebrates and invertebrates [3]. Methanogens can use a number of different substrates, such as hydrogen, formate, acetate, methanol, and methlyamines, to reduce carbon dioxide to methane during the normal fermentation of feed [4], and studies on ruminants have shown that the production of enteric methane results in loss of gross energy available to the host $[5,6]$. Methanogens have been isolated from various animals $[7,8]$ and several studies using culture-independent methods, including 16S rRNA gene clone library analysis, have provided some useful data on the diversity and abundance of methanogens in rumen [9-12]. In other hindgut fermenters, such as humans and pigs, the diversity and density of methanogens in the human colon were different among obese and lean, or post-gastric-bypass, individuals [13]. Moreover, the structure of fecal methanogens appears to differ among different pig breeds $[14,15]$. These studies indicated that methanogen diversity in the GIT may be host species-specific and, or, function-dependent. Therefore, we hypothesize that the methanogens present in the white rhinoceros may have a unique community structure and composition than those from other herbivores, which have been studied to date.

The objectives of the present study are to elucidate the molecular diversity and community structure of methanogens in the hindgut of the white rhinoceroses using $16 \mathrm{~S}$ rRNA gene clone library analysis.

\section{Methods}

\section{Sample sources and processing}

All animals were legally transported from South Africa into Yunnan Wild Animal Park in China as ornamental animals in July, 2010 under permission of the State Forestry Bureau of China, and were managed according to the guidelines of animal care and use approved by the Chinese Authority.

Seven adult white rhinoceroses (4 males and 3 females), aged from 6 to 8 years old, were selected as experimental animals. Feed consisted of pellets, apple, carrot, fresh forage/alfalfa and alfalfa hay with a ratio as 10:5:10:80:10. The ingredients and proportion of the pellet feed (per $100 \mathrm{~kg}$ ) were as follows: $30 \mathrm{~kg}$ maize, $20 \mathrm{~kg}$ soybean meal, $8 \mathrm{~kg}$ wheat bran, $8 \mathrm{~kg}$ wheat, $5 \mathrm{~kg}$ malt root, $3 \mathrm{~kg}$ rice bran, $12 \mathrm{~kg}$ alfalfa meal, $7 \mathrm{~kg}$ oil cake, $1.5 \mathrm{~kg}$ yeast extract, $1.5 \mathrm{~kg}$ bone meal, $1 \mathrm{~kg}$ salt, $1 \mathrm{~kg}$ fish meal, $0.1 \mathrm{~kg}$ compound vitamins, $0.1 \mathrm{~kg}$ lysine, $1.2 \mathrm{~kg}$ di-calcium phosphate, $0.1 \mathrm{~kg}$ sodium selenite-Vitamin $\mathrm{E}$, $0.7 \mathrm{~kg}$ calcium carbonate, $0.1 \mathrm{~kg}$ trace element, $0.1 \mathrm{~kg}$ zinc sulfate and $0.1 \mathrm{~kg}$ copper sulfate. Approximately $10 \mathrm{~g}$ of fresh feces were collected from each rhinoceros in August, 2012, and stored on ice in a sterilized 15-ml centrifuge tube until transported to the laboratory (approximately $2 \mathrm{~h}$ ). Fecal samples were then stored at $-20^{\circ} \mathrm{C}$ until further processing. The collection of the fecal samples and the subsequent analysis was permitted by Yunnan Wild Animal Park and the State Forestry Bureau of China.

\section{DNA extraction, PCR amplification and clone library construction}

Nucleic acids were extracted from $0.5 \mathrm{~g}$ of feces using the bead-beating method described by Zoetendal et al. [16], and DNA samples were purified with a PCR Clean-Up system (Promega, Madison, USA) and stored at $-20^{\circ} \mathrm{C}$.

Methanogen specific primers Met86F and Met1340R [17] were used to amplify archaeal $16 \mathrm{~S}$ rRNA genes. The amplification was initiated with a denaturation at $94^{\circ} \mathrm{C}$ for $3 \mathrm{~min}$, followed by 40 cycles of $94^{\circ} \mathrm{C}$ for $30 \mathrm{~s}, 58^{\circ} \mathrm{C}$ for $30 \mathrm{~s}$ and $72^{\circ} \mathrm{C}$ for $90 \mathrm{~s}$, and a last extension at $72^{\circ} \mathrm{C}$ for $10 \mathrm{~min}$. The PCR reaction mixture $(50 \mu \mathrm{l})$ consisted of $200 \mathrm{nM}$ of each primer, approximately $0.35 \mu \mathrm{g}$ of template DNA, $1 \times$ Taq reaction buffer, $200 \mu \mathrm{M}$ of each dNTP, $2 \mathrm{mM}$ of $\mathrm{MgCl}_{2}$ and four units of Taq DNA polymerase. The amplicons were purified using a PCR Clean-Up system (Promega, Madison,USA).

A 16S rRNA gene clone library was constructed using equal quantities of purified pooled PCR products from each animal, that had been cloned into the pGEM-T Easy vector and transformed into Escherichia coli TOP10 (Promega, Madison,USA). A total of 160 transformed clones with correct sized inserts were selected and confirmed by sequence analysis (Invitrogen, Shanghai, China).

Estimation of archaeal diversity and phylogenetic analysis Sequences were checked for chimeras using the chimera detection program BELLERPHON as part of the software package MOTHUR (ver 1.23.1). Based on a species-level sequence identity criterion of 98\% [18], MOTHUR was used to assign the $16 \mathrm{~S}$ rRNA gene sequences to

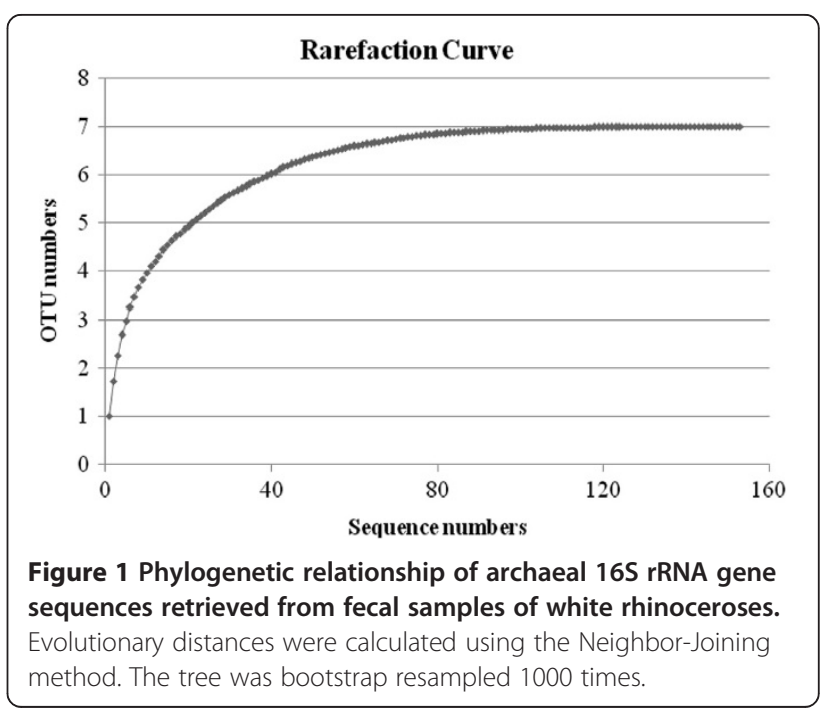


Table 1 Operational taxonomic units (OTUs) of archaeal 16S rRNA gene sequences from feces of white rhinoceroses

\begin{tabular}{|c|c|c|c|c|c|c|}
\hline OTU & phylotype & No. of sequences & Nearest valid taxon* & $\begin{array}{l}\% \text { Sequence } \\
\text { identity }\end{array}$ & $\begin{array}{l}\text { Nearest uncharacterized } \\
\text { clone }\end{array}$ & $\begin{array}{c}\text { \% Sequence } \\
\text { identity }\end{array}$ \\
\hline 1 & W-Rhino1 & 2 & Methanosphaera stadtmanae & 96.3 & HM573412 & 99.4 \\
\hline 1 & W-Rhino21 & 4 & Methanosphaera stadtmanae & 96.6 & HM573412 & 99.8 \\
\hline 2 & W-Rhino8 & 4 & Methanomassiliicoccus luminyensis & 88.1 & HM038364 & 98.6 \\
\hline 2 & W-Rhino22 & 2 & Methanomassiliicoccus luminyensis & 88.4 & HM038364 & 98.6 \\
\hline 3 & W-Rhino25 & 5 & Methanomassiliicoccus luminyensis & 87.8 & JN030604 & 95.9 \\
\hline 4 & W-Rhino33 & 3 & Methanomassiliicoccus luminyensis & 87.5 & JN030608 & 95.7 \\
\hline 5 & W-Rhino15 & 6 & Methanocorpusculum labreanum & 95.5 & AB739382 & 95.9 \\
\hline 5 & W-Rhino19 & 2 & Methanocorpusculum labreanum & 95.1 & AB739382 & 95.7 \\
\hline 5 & W-Rhino20 & 5 & Methanocorpusculum labreanum & 95.1 & AB739382 & 96.0 \\
\hline 5 & W-Rhino26 & 3 & Methanocorpusculum labreanum & 95.5 & AB739382 & 96.3 \\
\hline 5 & W-Rhino30 & 2 & Methanocorpusculum labreanum & 95.1 & AB739382 & 96.0 \\
\hline 5 & W-Rhino35 & 6 & Methanocorpusculum labreanum & 95.3 & AB739382 & 95.8 \\
\hline 5 & W-Rhino44 & 1 & Methanocorpusculum labreanum & 95.4 & AB739382 & 95.9 \\
\hline 5 & W-Rhino45 & 2 & Methanocorpusculum labreanum & 95.4 & AB739382 & 95.9 \\
\hline 6 & W-Rhino4 & 3 & Methanobrevibacter smithii & 97.3 & AB739317 & 98.9 \\
\hline 6 & W-Rhino7 & 5 & Methanobrevibacter smithii & 97.5 & AB739317 & 99.4 \\
\hline 6 & W-Rhino13 & 1 & Methanobrevibacter smithii & 97.6 & AB739317 & 99.6 \\
\hline 6 & W-Rhino16 & 7 & Methanobrevibacter smithii & 97.5 & AB739317 & 99.5 \\
\hline 6 & W-Rhino23 & 11 & Methanobrevibacter smithii & 97.5 & AB739317 & 99.4 \\
\hline 6 & W-Rhino28 & 4 & Methanobrevibacter smithii & 97 & AB739317 & 98.7 \\
\hline 6 & W-Rhino34 & 4 & Methanobrevibacter smithii & 97.5 & AB739317 & 99.5 \\
\hline 6 & W-Rhino36 & 1 & Methanobrevibacter smithii & 97.4 & AB739317 & 99.4 \\
\hline 6 & W-Rhino38 & 1 & Methanobrevibacter smithii & 97.5 & AB739317 & 99.4 \\
\hline 6 & W-Rhino39 & 1 & Methanobrevibacter smithii & 97.6 & AB739317 & 99.6 \\
\hline 6 & W-Rhino41 & 2 & Methanobrevibacter smithii & 97.4 & AB739317 & 99.3 \\
\hline 6 & W-Rhino42 & 1 & Methanobrevibacter smithii & 97.4 & AB739317 & 99.4 \\
\hline 6 & W-Rhino46 & 1 & Methanobrevibacter smithii & 97.5 & AB739317 & 99.4 \\
\hline 7 & W-Rhino2 & 3 & Methanocorpusculum labreanum & 95.4 & AB739382 & 96.2 \\
\hline 7 & W-Rhino3 & 1 & Methanocorpusculum labreanum & 95.4 & AB739382 & 96.2 \\
\hline 7 & W-Rhino5 & 5 & Methanocorpusculum labreanum & 95.2 & AB739382 & 96.2 \\
\hline 7 & W-Rhino6 & 9 & Methanocorpusculum labreanum & 95.2 & AB739382 & 95.7 \\
\hline 7 & W-Rhino9 & 4 & Methanocorpusculum labreanum & 95.4 & AB739382 & 96.2 \\
\hline 7 & W-Rhino10 & 1 & Methanocorpusculum labreanum & 95.4 & AB541926 & 96.0 \\
\hline 7 & W-Rhino11 & 3 & Methanocorpusculum labreanum & 95.1 & AB541926 & 95.8 \\
\hline 7 & W-Rhino12 & 7 & Methanocorpusculum labreanum & 95.1 & AB541926 & 95.6 \\
\hline 7 & W-Rhino14 & 2 & Methanocorpusculum labreanum & 95.2 & AB541926 & 95.8 \\
\hline 7 & W-Rhino17 & 2 & Methanocorpusculum labreanum & 95.1 & AB739382 & 95.9 \\
\hline 7 & W-Rhino18 & 1 & Methanocorpusculum labreanum & 95.3 & AB739382 & 96.1 \\
\hline 7 & W-Rhino24 & 2 & Methanocorpusculum labreanum & 95.4 & AB739382 & 96.2 \\
\hline 7 & W-Rhino27 & 1 & Methanocorpusculum labreanum & 95.6 & AB541926 & 96.0 \\
\hline 7 & W-Rhino29 & 7 & Methanocorpusculum labreanum & 95.3 & AB739382 & 96.1 \\
\hline 7 & W-Rhino31 & 1 & Methanocorpusculum labreanum & 95.3 & AB739382 & 96.1 \\
\hline 7 & W-Rhino32 & 2 & Methanocorpusculum labreanum & 96.2 & AB739400 & 96.9 \\
\hline
\end{tabular}


Table 1 Operational taxonomic units (OTUs) of archaeal 16S rRNA gene sequences from feces of white rhinoceroses (Continued)

\begin{tabular}{|c|c|c|c|c|c|c|}
\hline 7 & W-Rhino37 & 5 & Methanocorpusculum labreanum & 95.3 & AB739382 & 96.1 \\
\hline 7 & W-Rhino40 & 1 & Methanocorpusculum labreanum & 95.2 & AB739382 & 96.0 \\
\hline 7 & W-Rhino43 & 3 & Methanocorpusculum labreanum & 95.4 & AB739382 & 96.2 \\
\hline 7 & W-Rhino47 & 4 & Methanocorpusculum labreanum & 95.2 & AB739382 & 96.0 \\
\hline Totals & & 153 & & & & \\
\hline
\end{tabular}

*Nearest valid taxon with the same name means the same strain.

operational taxonomic units (OTUs). The sampling effort in the library for species-level OTUs was evaluated by calculating the coverage $(C)$ according to the equation $C=1$ - $(\mathrm{n} / \mathrm{N})$, where $\mathrm{n}$ is the number of OTUs represented by a single clone and $\mathrm{N}$ is the total number of clones analyzed in the library [19]. GenBank's Basic Local Alignment Search Tool (BLAST) [20] was used to presumptively identify the nearest validly described neighbor of each methanogen sequence. Lastly, a neighbor-joining tree was constructed using the phylogenetic software PHYLIP (ver 3.69) with 1,000 bootstrap resamplings of the dataset [21]. The nucleotide sequences reported in this paper have been deposited in the GenBank database under accession numbers JX833566 to JX833612.

\section{Results}

A total of 153 non-chimeric 16S rRNA gene sequences were obtained from fecal samples of seven white rhinoceroses. Examination of the 153 sequences revealed 47 different phylotypes (Figure 1), which were assigned to 7 OTUs based on a $98 \%$ sequence identity criterion (Table 1). The coverage of the clone library was $95.4 \%$, indicating the library was well sampled (Figure 2). The CHAO 1 OTU estimate was 7, and the Shannon Index was $1.47 \pm 0.13$. Six sequences (4\%) were assigned to OTU-1 and had $96.6 \%$ identity to Methanosphaera stadtmanae (Table 1). OTU-2 (6 sequences), OTU-3 (5 sequences) and OTU-4 (3 sequences) were distantly related to Methanomassiliicoccus luminyensis with sequences ranging from $87.5 \%$ to $88.4 \%$. OTU-5 (27 sequences) and OTU-7 (64 sequences) were related to Methanocorpusculum labreanum with sequence identities of $96.2 \%$ and $95.5 \%$, respectively. Forty-two sequences (27\%) were assigned to OTU-6 and had 97.3\% to $97.6 \%$ sequence identity to Methanobrevibacter smithii.

At the phylotype level, W-Rhino1 and W-Rhyno21 (both assigned to OTU-1) were closely related to an uncharacterized archaeal clone from pig feces (99.4\% and 99.8\% identities, respectively) [14] (Table 1, Figure 1). The two phylotypes belonging to OTU-2 had 98.6\% identity to an uncultured clone from bovine rumen [22] (Table 1, Figure 1). Two sequences were related to two methanogen clones (JN030604 and JN030608) from continental shelf of India with $96.0 \%$ and $95.7 \%$ identity, respectively (Table 1, Figure 1). Five sequences assigned to OTU-7 showed genus-level (95.6\% to $96 \%$ ) sequence identity to an uncharacterized clone from cattle manure [23], while the remaining phylotypes that were assigned to OTU-7 were related to a methanogen clone from the hindgut of the pony (AB739382) with 95.7\% to 96.9\% identities (Table 1, Figure 1). All phylotypes assigned to OTU-5 also showed genus-level (95.7 to 96.3\%) sequence identity to a clone from the hindgut of the pony (AB739382) (Table 1, Figure 1). The clone library OTU coverage rate was $95.4 \%$, indicating that the library was very well sampled for the diversity it contained.

Phylogenetic analysis indicated that all 47 phylotypes (i.e., 153 sequences) belonged to four monophyletic groups (Figures 1 and 3). Phylotypes assigned to OTU-5 and OTU-7 grouped together on a branch as the sister group to Methanocorpusculum labreanum with very strong bootstrap support (99\%), OTU-1 phylotypes grouped within the genus Methanosphaera, and phylotypes assigned to OTU-6 grouped together on a branch with several species belonging to the genus Methanobrevibacter. The remaining phylotypes grouped together with other uncultivated methanogens belonging to a recently proposed seventh order of methanogenic archaea, the Methanoplasmatales [24].

\section{Discussion}

To the best of our knowledge, the current study is the first to report methanogens closely related to Methanocorpusculum labreanum [25] as the predominant phylotype in the gastrointestinal tract of animals. This is in contrast to many other studies, where Methanobrevibacter species were the dominant methanogen phylotypes in other herbivores worldwide [26-30]. In the present study, approximately $60 \%$ of the $15316 \mathrm{~S}$ rRNA gene sequences obtained from the feces of white rhinoceroses was related to the genus Methanocorpusculum. However, it is important to note that the use of a pooled sample makes it impossible to know if these methanogens were prevalent in all seven animals. In contrast, the proportion of the sequences assigned to the genus Methanobrevibacter was only $27 \%$.

Studies on ruminants [10] and on monogastric animals, such as pigs and gnotobiotic mice [14,31], have indicated 


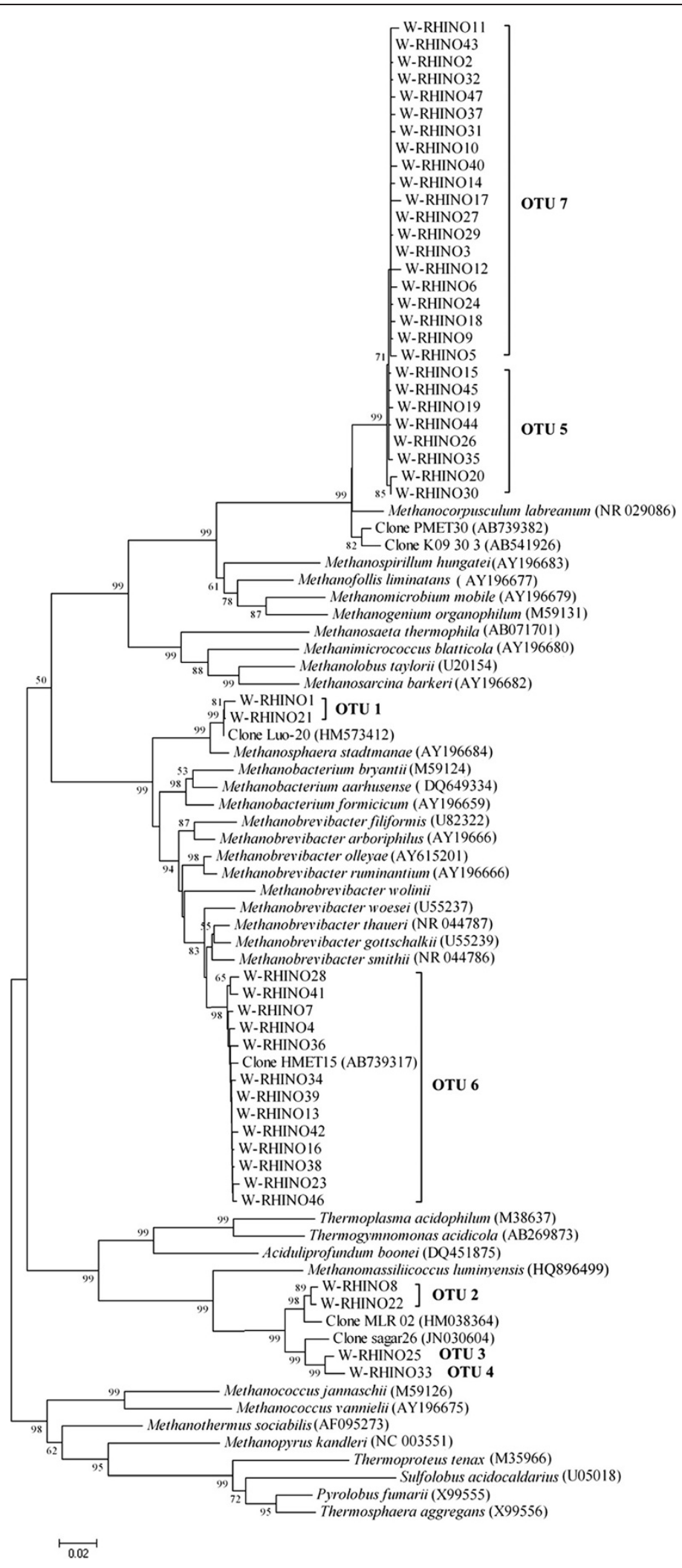

Figure 2 Rarefaction curve of the archaeal 16S rRNA clone library obtained from hindgut of the white rhinoceroses. 


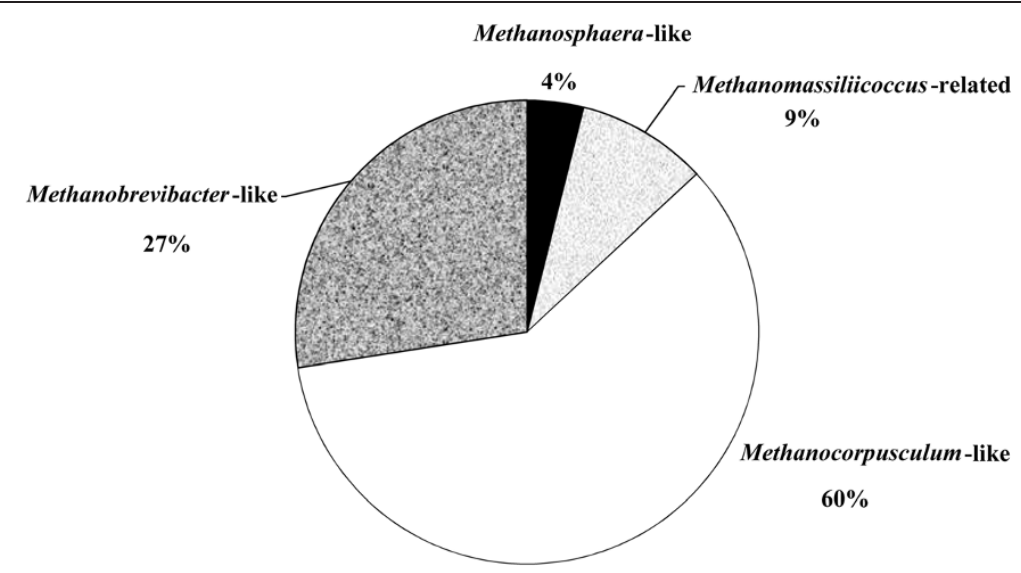

Figure 3 Pie chart representation of methanogen 16S rRNA gene clone distributions in feces of white rhinoceroses. Methanocorpusculum-like sequences represented the majority in the library (60\%), followed by Methanobrevibacter-like (27\%), Methanomassiliicoccus-related (9\%) and Methanosphaera-like (4\%).

that Methanobrevibacter smithii affects the efficiency of digestion of dietary polysaccharides, whereas most strains of Methanocorpusculum labreanum have been isolated from sediments, anaerobic digesters, waste water [32,33], and the hindgut of termites [34,35]. Methanocorpusculum labreanum also requires acetate as a carbon source and has additional complex nutritional requirements [36]. Termites, horses and very large herbivores such as rhinoceroses and elephants are typical hindgut fermenters [37]. The common distribution of Methanocorpusculum labreanum in the hindgut of termites and rhinoceroses may likely be due to the digestive physiology of the hindgut and may play an unusual function for digestion of dietary fibers.

Facey et al. [38] found that Methanosphaera stadtmanae, a methanol utilizer, was the predominant methanogen in the gastrointestinal tract of orangutans. The researchers suggested that the high prevalence of Methanosphaera stadtmanae may likely due to the increased availability of methanol from the highly frugivorous diet of the orangutans. Methanosphaera stadtmanae was also found in the current study, but was represented in only $4 \%$ of the total sequences. Like other species of Methanobrevibacter, Methanocorpusculum labreanum also produces methane from $\mathrm{H}_{2}-\mathrm{CO}_{2}$, or formate, but not from methanol and methylanmines [24]. Thus, we inferred that the low representation of Methanosphaera stadtmanae may be due to the predominant presence of Methanocorpusculum labreanum, or because of the small quantity of methanol produced by the fermentation of plant material in the hindgut of the white rhinoceroses, which needs to be further studied.

Based on calculations derived from in vitro studies and domestic ruminants, the growth of gut methanogens has been postulated to be a limiting factor in large herbivore digestive physiology [39]. For example, the relatively fast passage rates in elephants, the largest extant terrestrial mammal, have been interpreted in part as a countermeasure against the danger of disproportional methanogen growth [37]. However, for some smaller mammalian or reptilian herbivores, the food particle retention times surpass the 4-day threshold postulated by Van Soest (1994). In these species, the fermentation products are better absorbed and not available as substrate for slowgrowing methanogens. Therefore, we speculate that the particular species of methanogens found in the hindgut of the white rhinoceros may be well suited in these large herbivores and play an unique role during the fermentation of the plant materials. Further studies on the function of these methanogen species are needed.

In the present study, the majority of methanogen sequences showed a closer relationship to uncharacterized clones in the equine hindgut. W-Rhino8 (assigned to OTU-2) was closely related to a methanogenic clone from the hindgut of the horse. All phylotypes belonging to OTU-5 and 15 phylotypes from OTU-7 were also related (96.9\%) to an uncultured archaeal clone from the hindgut of a pony. In a previous study, the horse was identified as an appropriate model when designing diets for captive animals such as large hindgut fermenters, elephants or rhinoceroses [40]. It is also been reported that the Indian rhinoceros resembles the domestic horse in most digestive characteristics, despite the immense body size difference between the species [1]. Interestingly, rhinoceroses and horses are both odd-toed ungulates belonging to the order Perissodactyla. Thus, the closer phylogenetic relationship of methanogenic species between rhinoceroses and horses may be associated with the common characteristics of their GIT (i.e. microbial habitat).

Our library also uncovered some unidentified archaeal sequences belonging to OTU-2, OTU-3 and OTU-4. 
The sequences were only $87.8 \%$ to $88.4 \%$ similar to Methanomassiliicoccus luminyensis, a new methanogen recently isolated from human stool [41] and belonging to the newly proposed order Methanoplasmatales [24].

\section{Conclusions}

In conclusion, the white rhinoceros harbors a unique fecal community of methanogens distinct from other animals, but with more similarity to horses and ponies. Methanocorpusculum labreanum represents the predominant (60\%) methanogenic species in the hindgut of the white rhinoceros. A number of novel methanogen sequences were also found, but their functional role in the digestion and health of the white rhinoceros awaits further investigation.

\section{Availability of supporting data}

The data sets supporting the results of this article are included within the article.

\section{Competing interests}

The authors declare that they have no competing interests.

\section{Authors' contributions}

$\mathrm{YL}$ designed the study, carried out the sequence alignment and drafted the manuscript. ADGW participated in the sequence alignment and performed the statistical analysis. $\mathrm{YL}$ participated in the design of the study. $\mathrm{HL}$ participated in the sequence alignment. QY participated in the design of the study. $L L$ and MY helped to draft the manuscript. All authors read and approved the final manuscript.

\section{Acknowledgments}

This work was supported by Young Scientist Fund of Department of Education of Sichuan Province (112A081). The authors thank Yunnan Wilde Animals Park for the providing of the white rhinoceros.

\section{Author details}

${ }^{1}$ Key Laboratory for Animal Disease-Resistance Nutrition of China and Ministry of Education; Institute of Animal Nutrition, Sichuan Agricultural University, 625014, Ya'an, China. ²Department of Animal Science, University of Vermont, 570 Main Street, Burlington, Vermont 05405, USA. ${ }^{3}$ Yunnan Wilde Animals Park, 18 Fengyuan Road, Panlong District, 650218, Kunming, China. ${ }^{4}$ Yunnan Natural Forest Center, 80 Jinhun Road, Panlong District, 650224, Kunming, China. ${ }^{5}$ Yunnan Business Information Engineering School, Panlong District, 650204, Kunming, China. ${ }^{6}$ College of Animal Science and Technology, Sichuan Agricultural University, 625014, Ya'an, China.

Received: 28 January 2013 Accepted: 10 September 2013 Published: 12 September 2013

\section{References}

1. Clauss M, Polster C, Kienzle E, Wiesner H, Baumgartner K, Von Houwald F, Ortmann S, Streich WJ, Dierenfeld ES: Studies on digestive physiology and feed digestibilities in captive Indian rhinoceros (Rhinoceros unicornis). J Anim Physiol An N 2005, 89(3-6):229-237.

2. IUCN: International Union for Conservation of Nature and Natural Resources (IUCN) Red list of threatened species. 2012. http://www.iucnredlist.org/ details/4185/0

3. Hackstein JHP, van Alen TA: Fecal methanogens and vertebrate evolution. Evolution 1996, 50(2):559-572

4. Samuel BS, Gordon I: A humanized gnotobiotic mouse model of hostarchaeal-bacterial mutualism. P Natl Acad Sci USA 2006, 103(26):1001110016.

5. Johnson K, Johnson D: Methane emissions from cattle. J Anim Sci 1995, 73(8):2483-2492
6. Machmüller A, Ossowski D, Kreuzer M: Comparative evaluation of the effects of coconut oil, oilseeds and crystalline fat on methane release, digestion and energy balance in lambs. Anim Feed Sci Tech 2000, 85(1-2):41-60.

7. Miller TL, Wolin M: Methanogens in human and animal intestinal tracts. Syst Appl Microbiol 1986, 7(2-3):223-229.

8. Miller TL, Wolin M, Kusel E: Isolation and characterization of methanogens from animal feces. Syst Appl Microbiol 1986, 8(3):234-238.

9. Wright ADG, Williams AJ, Winder B, Christophersen CT, Rodgers SL, Smith KD: Molecular diversity of rumen methanogens from sheep in Western Australia. Appl Environ Microbiol 2004, 70(3):1263-1270.

10. Denman SE, Tomkins NW, McSweeney CS: Quantitation and diversity analysis of ruminal methanogenic populations in response to the antimethanogenic compound bromochloromethane. FEMS Microbiol Ecol 2007, 62(3):313-322.

11. Wright ADG, Auckland $C H$, Lynn DH: Molecular diversity of methanogens in feedlot cattle from Ontario and Prince Edward Island, Canada. Appl Environ Microbiol 2007, 73(13):4206-4210.

12. Pei CX, Mao SY, Cheng YF, Zhu WY: Diversity, abundance and novel $16 \mathrm{~S}$ rRNA gene sequences of methanogens in rumen liquid, solid and epithelium fractions of Jinnan cattle. Animal 2010, 4(1):20-29.

13. Zhang H, DiBaise JK, Zuccolo A, Kudrna D, Braidotti M, Yu Y, Parameswaran $P$, Crowell MD, Wing R, Rittmann BE: Human gut microbiota in obesity and after gastric bypass. Natl Acad Sci USA 2009, 106(7):2365-2370.

14. Luo Y, Su Y, Wright ADG, Zhang L, Smidt H, Zhu W: Lean breed Landrace pigs harbor fecal methanogens at higher diversity and density than obese breed Erhualian pigs. Archaea 2012:1-9. doi:10.1155/2012/605289.

15. Mao SY, Yang CF, Zhu WY: Phylogenetic analysis of methanogens in the pig feces. Curr Microbiol 2011, 62(5):1386-1389.

16. Zoetendal EG, Akkermans AD, De Vos WM: Temperature gradient gel electrophoresis analysis of $16 \mathrm{~S}$ rRNA from human fecal samples reveals stable and host-specific communities of active bacteria. Appl Environ Microbiol 1998, 64(10):3854-3859.

17. Wright ADG, Pimm C: Improved strategy for presumptive identification of methanogens using 165 riboprinting. J Microbiol Meth 2003, 55(2):337-349.

18. Wright ADG, Northwood KS, Obispo NE: Rumen-like methanogens identified from the crop of the folivorous South American bird, the hoatzin (Opisthocomus hoazin). ISME J 2009, 3(10):1120-1126.

19. Good IJ: The population frequencies of species and the estimation of population parameters. Biometrika 1953, 40(3-4):237-264.

20. Altschul SF, Madden TL, Schäffer AA, Zhang J, Zhang Z, Miller W, Lipman DJ: Gapped BLAST and PSI-BLAST: a new generation of protein database search programs. Nucleic Acids Res 1997, 25(17):3389-3402.

21. Felsenstein J: (Phylogeny inference package) documentation files. Version 3.62c. Seattle, Washington: Department of Genetics, University of Washington; 2004

22. Tan HY, Sieo CC, Lee CM, Abdullah N, Liang JB, Ho YW: Diversity of bovine rumen methanogens in vitro in the presence of condensed tannins, as determined by sequence analysis of $16 \mathrm{~S}$ rRNA gene library. J Microbiol 2011, 49(3):492-498

23. Yamamoto N, Asano R, Yoshii H, Otawa K, Nakai Y: Archaeal community dynamics and detection of ammonia-oxidizing archaea during composting of cattle manure using culture-independent DNA analysis. Appl Environ Microbiol 2011, 90(4):1501-1510.

24. Paul K, Nonoh JO, Mikulski L, Brune A: 'Methanoplasmatales': thermoplasmatales-related archaea in termite guts and other environments are the seventh order of methanogens. App/ Environ Microbio/ 2012. doi:10.1128/AEM.02193-12.

25. Anderson IJ, Siprawska-Lupa M, Goltsman E, Lapidus A, Copeland A, Glavina T, Rio D, Tice H, Dalin E, Barry K, Pitluck S, Hauser L, Land M, Luca S, Richardson P, Whitman WB, Kyripides NC: Complete genome sequence of Methanocorpusculum labreanum type strain Z. Stand Genomic Sci 2009, 1 (2):197-203.

26. Hook SE, Northwood KS, Wright ADG, McBride BW: Long-term monensin supplementation does not significantly affect the quantity or diversity of methanogens in the rumen of the lactating dairy cow. Appl Environ Microbiol 2009, 75(2):374-380

27. Irbis C, Ushida K: Detection of methanogens and Proteobacteria from a single cell of rumen ciliate protozoa. J Gen Appl Microbiol 2004, 50(4):203-212 
28. Ouwerkerk D, Turner A, Klieve A: Diversity of methanogens in ruminants in Queensland. Anim Prod Sci 2008, 48(7):722-725.

29. Whitford MF, Teather RM, Forster RJ: Phylogenetic analysis of methanogens from the bovine rumen. BMC Microbio/ 2001, 1(1):5.

30. Wright ADG, Ma X, Obispo NE: Methanobrevibacter phylotypes are the dominant methanogens in sheep from Venezuela. Microbial Ecol 2008, 56(2):390-394

31. Samuel BS, Gordon Jl: A humanized gnotobiotic mouse model of host-archaeal-bacterial mutualism. Proc Natl Acad Sci USA 2006, 103(26):10011-10016.

32. Zhao Y, Boone DR, Mah RA, Boone JE, Xun L: Isolation and characterization of Methanocorpusculum labreanum sp. nov. from the LaBrea Tar Pits. Int J Syst Bacteriol 1989, 39(1):10-13.

33. Garcia JL, Ollivier B, Whitman WB: The order Methanomicrobiales. Prokaryotes 2006, 3:208-230.

34. Ohkuma M, Noda S, Horikoshi K, Kudo T: Phylogeny of symbiotic methanogens in the gut of the termite Reticulitermes speratus. FEMS microbiol lett 2006, 134(1):45-50.

35. Purdy KJ: The distribution and diversity of Euryarchaeota in termite guts. Adv Appl Microbiol 2007, 62:63-80.

36. Barber RD: Methanogenesis: ecology. New York: John Wiley \& Sons; 2007. doi:10.1002/9780470015902.a0000475.pub2.

37. Clauss M, Frey R, Kiefer B, Lechner-Doll M, Loehlein W, Polster C, Rössner G, Streich WJ: The maximum attainable body size of herbivorous mammals: morphophysiological constraints on foregut, and adaptations of hindgut fermenters. Oecologia 2003, 136(1):14-27.

38. Facey HV, Northwood KS, Wright ADG: Molecular Diversity of methanogens in fecal samples from captive Sumatran orangutans (pongo abelii). Amer J Primatol 2012, 74(5):460-468.

39. Hofmann R: Evolutionary steps of ecophysiological adaptation and diversification of ruminants: a comparative view of their digestive system. Oecologia 1989, 78(4):443-457.

40. Oftedal OT, Baer DJ, Allen ME: The feeding and nutrition of herbivores Chicago (USA): University of Chicago Press; 1996

41. Dridi B, Fardeau ML, Ollivier B, Raoult D, Drancourt M: Methanomassiliicoccus luminyensis gen. nov., sp. nov., a methanogenic archaeon isolated from human faeces. Int I Syst Evol Microbiol 2012, 62(Pt 8):1902-1907.

doi:10.1186/1471-2180-13-207

Cite this article as: Luo et al:: Diversity of methanogens in the hindgut of captive white rhinoceroses, Ceratotherium simum. BMC Microbiology 2013 13:207

\section{Submit your next manuscript to BioMed Central and take full advantage of:}

- Convenient online submission

- Thorough peer review

- No space constraints or color figure charges

- Immediate publication on acceptance

- Inclusion in PubMed, CAS, Scopus and Google Scholar

- Research which is freely available for redistribution 